\title{
Bonusprogramme - Zwischen Wettbewerb und Prävention
}

\section{Eine Bestandsaufnahme über die Ausgestaltung präven- tiver Bonusprogramme}

\begin{abstract}
Bonusprogramme der gesetzlichen Krankenversicherungen (GKV) werden eingesetzt, um das gesundheitsbewusste Verhalten der Versicherten mithilfe von (materiellen) Anreizen (Boni) positiv zu beeinflussen bzw. zu bestätigen. Ziel ist es, durch die positiven Verhaltensänderungen - insbesondere (Hoch-)Risikogruppen - unausgeschöpfte präventive Rationalisierungspotenziale auszuschöpfen. Erste Studien deuten darauf hin, dass Mitnahmeeffekte vor Präventionseffekte dominieren. Im Fokus des Artikels steht die Darstellung der Gestaltungselemente und der aktuellen Justierung von Bonusmodellen, um dieser Tendenz nachzugehen.
\end{abstract}

\section{Scherenberg, W. Greiner}

\section{Hintergrund}

Einen regelrechten Boom erfuhren Bonusprogramme auf Wirtschaftsebene in Deutschland mit dem Fall des Rabattgesetzes und der Zugabeverordnung im Sommer 2001.[1] Angesichts der rasanten Verbreitung seit der Niederlegung der gesetzlichen Restriktionen, gemessen an den angebotenen Programmen und den hohen Mitgliederzahlen, lag es nahe, die positiven Anreizeffekte des Instrumentariums auch für die Zwecke des Gesundheitswesens nutzbar zu machen. So verschaffte der Gesetzgeber mit der Einführung des Gesundheitsmodernisierungsgesetzes (GMG) zum 01.01.2004 auch für die GKVn die Möglichkeit, ihren Kunden einen Bonus für gesundheitsbewusstes Verhalten zu offerieren. [2] Während Bonusprogramme auf Wirtschaftsebene in erster Linie auf Neukundengewinnung und Kundenbindung abzielen, sollen präventive Bonusprogramme das gesundheitsbewusstes Verhalten der Versicherten fördern und den Wettbewerb um die beste gesundheitliche Versorgung stärken. Als integraler Bestandteil des Leistungskatalogs der GKVn sollen Leistungen zur Primärprävention „den

V. Scherenberg, Europäische Institut für Angewandtes Kundenmanagement (eifk) und W. Greiner, Universität Bielefeld, Fakultät für Gesundheitswissenschaften, Gesundheitsökonomie und Gesundheitsmanagement allgemeinen Gesundheitszustand verbessern und insbesondere einen Beitrag zur Verminderung sozial bedingter Ungleichheiten von Gesundheitschancen erbringen ".[3] Die Problematik der sozial bedingten Ungleichheiten von Gesundheitsrisiken läuft Gefahr, sich aufgrund des immer weiter auseinanderklaffenden Gesundheitsverhalten zwischen Arm und Reich zu verstärken. Erneut konnte der 2. Armuts- und Reichtumsbericht des Robert Koch Institutes bestätigen, dass vor allem die sozioökonomische Lage einen wesentlichen Einfluss auf das Gesundheitsverhalten und auf den gesundheitlichen Status der Versicherten hat.[4] Folglich wird der größte Veränderungsbedarf zur Ausschöpfung des präventiven Rationalisierungspotenzials in der Überwindung des Präventionsdilemmas, sprich in der verstärkten Erreichung von sozial benachteiligten und damit gesundheitlich vulnerabler Gruppen, gesehen.[5] In Anbetracht dieser Erkenntnis gewinnen Anreiz- und Steuerungsinstrumente zur Angleichung sozioökonomischer Gesundheitsund Mortalitätsunterschiede immer mehr an Bedeutung. Mangelnde Erfahrungen und Studien führen dazu, dass Bonusprogramme im öffentlichen Diskus mitunter als „Marketinggag“[6] und nicht als „Präventionsinstrument mit Mehrwert" tituliert werden. Trotz der Zunahme empirischer Erkenntnisse über die positiven präventiven Wirkungseffekte (bspw. „BKK-Studie zur Effizienz von Bonusprogrammen“)[7], schürt die Unsicherheit über die Refinanzierung der Ausschüttungen durch langfristige 


\section{THEMA}

Einsparungen die aktuelle Kosten-Nutzen-Diskussion. Zudem eignen sich trotz des geforderten Effizienznachweises Bonusprogramme prinzipiell auch zur Neukundengewinnung, Kundenbindung[8] und Risikoselektion. Eine indirekte Risikoselektion liegt dann vor, wenn die Angebote so justiert werden, dass sie für schlechte Risiken unattraktiv (z. B. durch zu hoch angesetzte Mindestanforderung) und für gute Risiken interessant erscheinen. [9] Um dieser Option einen Riegel vorzuschieben, hat der Gesetzgeber die gesetzliche Verpflichtung auferlegt, dass Aufwendungen lt. $\S 65$ a Abs. 4 SGB V für Bonusprogramme mittelfristig aus Ersparnissen und Effizienzreserven stammen müssen.[10] Wiederum sind die Kassen angesichts des ökonomischen Drucks mehr den je gefordert im zunehmenden Verdrängungswettbewerb auch kurzfristig erreichbare wirtschaftliche Ziele ins Blickfeld zu nehmen. Die gesetzlich verankerten Grundmaximen der Sozialversicherung und der gesetzliche Auftrage der GKVn[11] zwingt die Kassen, die sozialen Ziele mit den eigenen Unternehmens- und Marketingzielen in Einklang zu bringen. Bei der Programminitiierung stellt die Kassen folglich vor dem Dilemma die bestehenden Zielkonflikte miteinander zu vereinen. Je nach kommerziell oder präventive Ausrichtung sind, lassen sich folgende Ziele verwirklichen (siehe Tabelle 1)[12]:

Zwar orientieren sich die derzeit umgesetzten Bonusmodelle am gesetzlichen Rahmen, die individuelle Ausgestaltung hängt jedoch von der konzeptionellen Kreativität der einzelnen Kasse ab. Während zum § 20 SGB $\mathrm{V}$ von den Spitzenverbänden der Krankenkassen prioritäre Handlungsfelder im „Leitfaden Prävention“ niedergelegt wurden,[13] existieren für die Umsetzung des § 65a SGB V bis dato keine spezifischen Qualitätsanforderungen[8] oder freiwillige Leitlinien (auf Verbandsebene). Anreizsysteme können als hochkomplexe Gebilde bezeichnet werden, die eine Vielzahl an Gestaltungsmöglichkeiten bieten.
Das Anliegen der Forschungsarbeit war es daher, mögliche Optimierungspotenziale im Sinne einer Verbesserung der präventiven Gesundheit durch eine Transparenz der umfangreichen Programmsystematik präventiver Bonusprogramme zu identifizieren.

\section{Methodik}

Die Grundlage der quantitativen und qualitativen Bestandsaufnahme (siehe Tabelle 2; Zeitraum: März bis Mai 2007) bildete ein vom Bundesversicherungsamt zur Verfügung gestellter Datensatz ( $\mathrm{n}=241$, Datenselektion vom 27.02.2007) der am Markt existierenden gesetzlichen Krankenkassen. Als Einschlusskriterien gelten alle Bonusprogramme, die nach $\S 65 a$ Abs. 1 SGB V initiiert wurden. Ausgeschlossen wurden alle Bonusmodelle, die sich nicht am o. g. Paragrafen orientieren (z. B. DMP oder betriebliche Gesundheitsförderung) oder als Teilnahmeprämisse eine Koppelung mit anderen Instrumenten (z. B. Selbstbehalt wie der AOK-Bonustarif der AOK Brandenburg) vorsahen. Lediglich 17 Kassen (vorwiegend betriebsbezogene BKKn) konnten online nicht erreicht werden. Da die Untersuchung sich nicht auf die Kassen, sondern auf die unterschiedlichen Gestaltungselemente der Bonusmodelle konzentrierte, wurden Verbundprogramme (z. B. das AOK Prämienprogramm und das Bonusprogramm der LKKn) aufgrund einer möglichen Verzerrung der Ergebnisse nur einmalig berücksichtigt. Aus Verlässlichkeitsgründen wurde für die qualitative Erhebung in erster Linie auf Informationen der Satzungen der jeweiligen Kassen zurückgegriffen, während für die qualitative Analyse die Homepage-Inhalte (sowie Flyer) höher ins Gewicht fielen. In Anbetracht der Tatsache, dass ein Großteil der Bonusprogramme mit Einführung des GMGs ins Leben

Tabelle 1: Zielsetzungen präventiver und kommerzieller Bonusprogramme

\begin{tabular}{|c|c|c|}
\hline & Präventionsinstrument & Marketinginstrument \\
\hline $\begin{array}{l}\text { Kunden- } \\
\text { ebene }\end{array}$ & $\begin{array}{l}\rightarrow \text { Förderung des Gesundheitsbewusstseins } \\
\rightarrow \text { Erhöhung der Eigenverantwortung } \\
\rightarrow \text { Verringerung gesundheitsschädigender } \\
\quad \text { Verhaltensweisen } \\
\rightarrow \text { Steigerung der Lebensqualität } \\
\rightarrow \text { Honorierung gesundheitsförderlicher Lebensweisen }\end{array}$ & $\begin{array}{l}\rightarrow \text { Steigerung der Kundenzufriedenheit } \\
\rightarrow \text { Honorierung der Kundenloyalität durch } \\
\quad \rightarrow \text { spezielle Produktangebote } \\
\quad \rightarrow \text { Sonder- und Treuerabatte } \\
\quad \rightarrow \text { Privilegien und Sonderstati } \\
\rightarrow \text { Erhöhung des Kundennutzens }\end{array}$ \\
\hline $\begin{array}{c}\text { Kassen- } \\
\text { ebene }\end{array}$ & $\begin{array}{l}\rightarrow \text { Erhöhung der Inanspruchnahme von } \\
\text { Früherkennungsmaßnahmen } \\
\rightarrow \text { Anregung zu gesundheitsförderlichen } \\
\text { Verhaltensweisen (bzw. Verhaltensmodifikation) } \\
\rightarrow \text { Senkung und Vermeidung der } \\
\text { Schadenswahrscheinlichkeit (bzw. der } \\
\text { Schadenshöhe) } \\
\rightarrow \text { Erzielung von Einsparungen (Kostenreduktion) } \\
\rightarrow \text { Verminderung von Chancenungleichheiten }\end{array}$ & $\begin{array}{l}\rightarrow \text { Aktive Risikoselektion und Wettbewerbsabwehr } \\
\rightarrow \text { Realisierung von Kundenbindung durch die } \\
\text { Schaffung von Wechselbarrieren } \\
\rightarrow \text { Möglichkeit der Neukundengewinnung } \\
\rightarrow \text { Ausschöpfung von Cross-Selling-Potenzialen } \\
\quad \text { (Zusatzversicherungen usw.) } \\
\rightarrow \text { Sammlung von Marketinginformationen } \\
\rightarrow \text { Effizienzsteigerung }\end{array}$ \\
\hline
\end{tabular}

Quelle: Eigene Darstellung 
Tabelle 2: Übersicht identifizierter Bonusprogramme nach Kassenart und Kassenöffnung

\begin{tabular}{|c|c|c|c|c|c|}
\hline Kassenöffnung & Kassenart & Gesamt & ja & nein & $\begin{array}{c}\text { keine } \\
\text { Internetpräsenz }\end{array}$ \\
\hline \multirow[t]{6}{*}{ geöffnet } & AOK & $8(16)$ & 6 & 2 & \\
\hline & EKArb & 3 & 3 & & \\
\hline & EKAng & 7 & 6 & 1 & \\
\hline & IKK & 16 & 13 & 3 & \\
\hline & BKK & 131 & 104 & 27 & 4 \\
\hline & $\mathrm{BKn}$ & 1 & 1 & & \\
\hline \multirow[t]{2}{*}{ geschlossen } & LKK & $1(9)$ & 1 & & \\
\hline & SeeK & 1 & 1 & & \\
\hline \multirow[t]{2}{*}{ betriebsbezogen } & BKK & 40 & 22 & 18 & 13 \\
\hline & Summe & $225(241)$ & 157 & 51 & 17 \\
\hline
\end{tabular}

Quelle: Eigene Darstellung

Abbildung 1: Kategorisierung der Bonifizierungskriterien

\begin{tabular}{|c|c|c|c|}
\hline \multirow{3}{*}{$\begin{array}{l}\text { (1) } \\
\begin{array}{c}\text { Bonifizierungs- } \\
\text { kriterien }\end{array}\end{array}$} & \multicolumn{3}{|c|}{$\begin{array}{l}\text { (1.1) Marketingstrategische Bonifizierungskriterien } \\
\text { (Mitgliederwerbung, Eintrittsanreize etc.) }\end{array}$} \\
\hline & \multirow{2}{*}{$\begin{array}{l}(1.2) \\
\text { Gesundheits- } \\
\text { bezogene } \\
\text { Bonifizierungs- } \\
\text { kriterien }\end{array}$} & $\begin{array}{l}\text { Verhaltensorientierte } \\
\text { Anforderungen } \\
\text { ("Input-Kriterien") }\end{array}$ & $\begin{array}{l}\text { Belohnung von Aktivitäten } \\
\rightarrow \text { dauerhafte (z. B. Sportkurse) } \\
\rightarrow \text { einmalige (z. B. Check-ups nach § } 25 \text { SGB V) }\end{array}$ \\
\hline & & $\begin{array}{l}\text { Ergebnisorientierte } \\
\text { Anforderungen } \\
\text { ("Output-Kriterien") }\end{array}$ & $\begin{array}{l}\text { Belohnung von Statuszuständen } \\
\rightarrow \text { ergebnisorientiert (z. B. BMI) } \\
\rightarrow \text { verhaltensorientiert (z. B. Impfpass) }\end{array}$ \\
\hline \multirow{6}{*}{$\begin{array}{l}\text { (2) } \\
\text { Bonifizierungs- } \\
\text { modalitäten }\end{array}$} & \multicolumn{3}{|c|}{ (2.1) Wahrscheinlichkeitsdimension (Schwierigkeitsgrad der Anforderungen) } \\
\hline & \multirow{2}{*}{$\begin{array}{c}(\mathbf{2 . 2}) \\
\text { Zeitdimension }\end{array}$} & $\begin{array}{l}\text { Bonifizierung nach } \\
\text { Zeitintervallen }\end{array}$ & $\begin{array}{l}\rightarrow \text { Zeitpunktbezogene Bonifizierung } \\
\rightarrow \text { Zeitraumbezogene Bonifizierung }\end{array}$ \\
\hline & & $\begin{array}{c}\text { Bonifizierung nach } \\
\text { Anforderungserreichung }\end{array}$ & $\begin{array}{l}\rightarrow \text { Bonifizierung von Mindestanforderungen } \\
\rightarrow \text { Bonifizierung von Einzelanforderungen }\end{array}$ \\
\hline & \multirow{2}{*}{$\begin{array}{l}\text { (2.3) Größen- } \\
\text { dimension } \\
\text { (langfristig) }\end{array}$} & $\begin{array}{l}\text { Steigerung außerhalb des } \\
\text { Abrechnungszeitraums }\end{array}$ & $\rightarrow$ gestaffelte Steigerungskomponenten \\
\hline & & $\begin{array}{l}\text { Steigerung innerhalb des Abrech- } \\
\text { nungszeitraums }\end{array}$ & $\begin{array}{l}\rightarrow \text { relative Steigerungskomponenten } \\
\rightarrow \text { absolute Steigerungskomponenten }\end{array}$ \\
\hline & \multicolumn{3}{|c|}{ (2.4) Größendimension (kurzfristig) (Gewichtung der einzelnen Anforderungen) } \\
\hline \multirow{3}{*}{$\begin{array}{c}\text { (3) } \\
\text { Bonifizierung }\end{array}$} & \multicolumn{2}{|c|}{ (3.1) direkte finanzielle Prämien } & $\rightarrow$ Geldprämie (direkte Geldzuwendungen) \\
\hline & \multicolumn{2}{|c|}{ (3.2) indirekte finanzielle Prämien } & $\begin{array}{l}\rightarrow \text { Geldwerte Prämien (Gutscheine usw.) } \\
\rightarrow \text { Erlebnisprämien (Events, Reisen etc.) }\end{array}$ \\
\hline & \multicolumn{2}{|c|}{ (3.3) Sachprämien } & $\rightarrow$ Sachprämien (Bücher, Sportgeräte etc.) \\
\hline
\end{tabular}

Quelle: Eigene Darstellung 


\section{THEMA}

gerufen wurde, überschneidet sich die Analyse mit der 3-jährigen Evaluationspflicht gemäß § 65a Abs. 3 SGB V. Es ist zu vermuten, dass die Kassen aufgrund der Evaluationsergebnisse aktuell Nachjustierungen ihrer Programme vornehmen. Angesichts der Dynamik des Marktes (Fusion sowie neue Instrumente, wie z. B. Wahltarife) können die qualitativen Ergebnisse der Untersuchung nur Tendenz beschreiben. Die Erkenntnisse der qualitativen Analyse bzw. der Identifizierung der spezifischen Gestaltungselemente (siehe Abbildung 1)[12] können wiederum dazu beitragen, Bonusmodelle zukünftig leichter zu analysieren, zu bewerten und zu optimieren.

\section{Ergebnisse: Aktuelle Ausgestaltung präventiver Bonusprogramme}

In Folge der hohen Zahl identifizierter Programme ( $\mathrm{n}=157)$ kann konstatiert werden, dass Bonusmodelle im Gesundheitswesen einer hohen Verbreitung unterliegen. Die Analyse zeigt, dass die Kassen bei der Ausgestaltung der ermittelten Stellhebel sehr unterschiedlich vorgehen. Die zentralen Ergebnisse der erfolgten Bestandsanalyse orientierte sich am dreiteiligen Grundgerüst des kossbielschen Effizienzmodells für Anreizsysteme: (1.) Kriterien (Bonifizierungsgrundlagen), (2.) Kriterien-Anreiz-Relation (Relationsvorschriften) und (3.) Anreize (Bonifizierungen)[14] und lassen sich wie folgt zusammenfassen:

\section{Hintergrïnde und Erkenntnisse hinsichtlich} der Bonifzierungskriterien: Voraussetzung für die Wirksamkeit präventiver Bonusprogramme ist die Beeinflussbarkeit der Ergebnisgrößen (Bonifizierung) durch die Versicherten. Grundsätzlich können Bemessungsgrundlagen für ein mess- und überprüfbares Verhalten input- wie auch outputorientiert erfolgen.[15] Im Kontext präventiver Bonusprogramme können diese als verhaltensorientierte (Input-Kriterien) und ergebnisorientierte (Output-Kriterien) Anforderungen identifiziert werden. Verhaltensorientierte Anforderungskriterien beziehen sich auf verhaltensorientierte qualitative Maßgrößen (wie z. B. die Teilnahme an einem Raucherentwöhnungskurs), die unabhängig von der Ergebnisdimension der Aktivität (z. B. Raucherstatus) honoriert werden. Eine weitere Abgrenzung kann nach dem zeitlichen Horizont erfolgen, sprich in der Belohnung langfristiger (z. B. Sportkurse) und einmaliger Aktivitäten (Check-ups etc.). Ergebnisorientierte Anforderungen hingegen berücksichtigen eher quantitative Maßgrößen bzw. eine „mögliche“ Erfolgsdimension der gesundheitlichen Verhaltensaktivitäten. Ein erfolgsorientierter Maßstab ist bspw. der Body-Mass-Index (BMI), während der Impfstatus als verhaltensorientierter Maßstab bezeichnet werden kann. Als Anforderungskriterien verwenden ca. die Hälfte der (eher größeren) Kassen ausschließlich ergebnisunabhängige Kriterien (z. B. Sport) als Bonifizierungsgrundlage. Kleinere Kassen tendieren dazu, zusätzlich ergebnisabhängige Komponenten (z. B. BMI) in ihren Anforderungskatalog mit einzubeziehen. Eine Belohnung positiver Entwicklungen im Gesundheitsverhalten findet fast gar nicht statt. Eine Ausnahme stellt das Healthmiles-Programm der BKK SECURVITA dar, dass neben dem BMI-Status (zwischen 18 und 27 Punkte) auch positive Gewichtsentwicklungen in Form einer Wertannäherung (um 2 Punkte innerhalb der letzten 12 Monate) honoriert. Mindestanforderungskriterien ohne Zusatzhonorierung können demotivierende Effekte auslösen, da alle über das geforderte Maß erbrachten Aktivitäten keine Honorierung erfahren. Knapp 9\% der Programme wurden selektiv auf spezifische Zielgruppen ausgerichtet. Merkmale, die bei der Ausrichtung berücksichtigt werden, sind neben dem Alter, der Familienstand und im geringen Maße das Geschlecht. Nur wenige Programme (bspw. der GEK) bieten kombinierte geschlechts- und altersspezifische Bonifizierungskriterien im Bereich der Früherkennung (z. B. mithilfe spezifischer Bonus-Scheckhefte). Die besonderen Bedürfnisse sozial benachteiligter Gruppen (z. B. Arbeitslose, Alleinerziehende, Migranten und Behinderte) wurden nur mangelhaft berücksichtigt. Eine Ausnahme stellt bspw. das DAK-Bonusprogramm dar. Während die auf das Merkmal „Familienstand“ ausgerichteter Programme eine Differenzierung auf „Singles“ und „Familien“ vorsehen, widmet sich die DAK darüber hinaus der Zielgruppe der Alleinerziehenden. Anforderungen (bzw. Bonifizierungen) können sowohl individuell als auch kollektiv erbracht werden. Die Stimulierung gesundheitlicher Aktivitäten auf der Mehr-Personen-Ebene beschränkt sich vorwiegend auf das nächste familiäre Umfeld.

2.) Erkenntnisse hinsichtlich der Kriteriums-Anreiz-Relation: Die Größendimension, sprich die Anreizintensität variiert je nach Programmsystematik der Kassen stark zeit- und größenabhängig. Die Größendimension beantwortet die Fragen, wie (z. B. stetig oder ansteigend) und wie stark die Bonifizierungen auf Veränderungen der Kriteriumsausprägung reagiert.[14] Ein Fünftel der Programme weist steigende Anreizkomponenten mit überwiegend langfristiger Ausrichtung auf. Die Erbringung und Bonifizierung erfolgt bei 70,2\% der Programme zu fest gesetzten Zeitpunkten bzw. im Jahresrhythmus. Andere Programme orientieren sich an der Erreichung bestimmter Mindestanforderungen (25,6\%), während 3.8\% der Modelle sich auf die Honorierung von Einzelaktivitäten konzentrieren. Das favorisierte Sammel- bzw. Identifikationsinstrument für die mitunter individuell kreierten Werteinheiten (Healthmiles, Powerpoints, GutLebenBonus, Gutfühlsternchen etc.) ist das klassische Bonusheft (82,2\%), gefolgt von Mitgliedschaftsprogrammen $(9,6 \%)$, online-gestützen Punktesystemen (3,8\%) und Einzelbonusanträgen (3,8\%). Die Entanonymisierung der Teilnehmer zur kommunikativen Unterstützung bei der Erfüllung der Anforderungen (z. B. Reminder) scheint aktuell nur rudimentär von Bedeutung.

3.) Erkenntnisse hinsichtlich der Anreize: Die Honorierung der teilnehmenden Versicherten findet mehrheitlich über reine Geldprämien (bzw. Erstattungen) $(62,4 \%)$ statt. Alternativ zu den Geldprämien offerieren einige Kassen ihren Versicherten Sachprämien (21\%), während sich ein geringer Teil (10,8\%) der Modelle ausschließlich auf die Belohnung von Sachanreizen konzentriert. Der Großteil der angebotenen Sachprämien weist eine Gesundheitsaffinität auf. Gesundheitsleistungen (wie z. B. Zusatzversicherungen oder IGel-Leistungen), die zur Reduktion des Präventionsdilemmas bei gleichzeitiger Steigerung des 
Sicherheitsbedürfnisses (wie Stabilität, Geborgenheit oder Schutz) beitragen können, stellen aktuell mit ca. 6\% die Seltenheit dar. Auch umfangreiche Prämienkataloge enthalten nur selten reine Gesundheitsleistungen. Hier wird versucht, in erster Linie durch eine Vielfalt an Prämien (oft mit Marken- und Eventcharakter) das Interesse der Versicherten zu wecken. Der Umfang der Anreizauswahl steigt tendenziell mit der Größe der potenziellen Zielgruppenerreichung des jeweiligen Programms.

\section{4.) Erkenntnisse hinsichtlich des Präventions-} dilemmas: Da sich die Elemente je nach Ausgestaltung untereinander stark beeinflussen können, sind spezifische Aussagen über die motivationalen Wirkungen der einzelnen Gestaltungshebel nicht möglich. Dennoch hat die Bestandsaufnahme gezeigt, dass von jedem identifizierten Gestaltungselement ein nicht zu unterschätzender Einfluss auf die Versicherten und damit auf die Zielerreichung der Kassen ausgehen kann. Angesichts der gesammelten Erkenntnisse kann konstatiert werden, dass zur Erreichung sozialer Ziele Programme verstärkt auf die Bedürfnisse von sozial benachteiligten Gruppen ausgerichtet werden müssten. Auch die Rücksichtnahme geschlechts- und altersdifferierende Unterschiede im Gesundheitsverhalten und im Gesundheitszustand besteht akuter Handlungsbedarf. Die Tabelle 3 bietet einen exemplarischen Überblick über die Beeinflussbarkeit der unterschiedlichen Stellhebel, je nachdem, ob das Programm eher marketingstrategische oder präventive Ziele verfolgt)[121:
Der Vergleich zeigt, dass je nach Gestaltung eine bewusste Beeinflussung beider Zielebenen möglich ist. Bei näherer Betrachtung wird deutlich, dass Zielkonflikte nicht zwangsläufig entstehen müssen. Mitunter können präventive und marketingstrategische Aspekte, wie bspw. die Stimulation von kollektiven Gesundheitsaktivitäten oder die Berücksichtigung positiver Gesundheitsentwicklungen, beide Zielebenen tangieren. Eine Vernachlässigung sozialer Ziele kann sich nicht nur negativ auf die wahrgenommene Gerechtigkeit (und Teilnehmermotivation), sondern auch negativ auf das Kassenimage auswirken. Ausgehend von der Tatsache, dass Kassen immer auch über schlechte Risiken verfügen und Kunden neueren Maßnahmen generell kritischer gegenüberstehen, wären die Kassen gut beraten, wenn sie ihre Ziele mit der Wahrnehmung der sozialen Gemeinschaftsaufgabe in Einklang brächten. Als zentrale Steuerungsgröße der Programme sollte verstärkt das Präventionspotenzial (der unterschiedlichen Bedarfs- bzw. Risikogruppen) herangezogen werden. Denn eine undifferenzierte Verfahrensweise ohne Fokussierung bedeutet angesichts hoher Streuverluste in vielen Fällen Ressourcenverschwendung. Insbesondere die Anforderungserfüllung sollte daran gekoppelt werden, inwieweit die Bonifizierungskriterien[12]

1.) von allen Versicherungsgruppen (je nach Alter, Geschlecht und sozialer Lage)

überhaupt erfüllbar sind und

Tabelle 3: Stellhebel für präventive und kommerzielle Ausrichtungen der Bonusprogramme

\begin{tabular}{|c|c|c|}
\hline & Präventionsinstrument & Marketinginstrument \\
\hline Kriterien & $\begin{array}{l}\rightarrow \text { Bonifizierung verhaltensorientierter Kriterien und } \\
\text { Bonifizierung von positiven Entwicklungen } \\
\rightarrow \text { Auswahl der Kriterien an gesundheitlichen } \\
\text { Verhaltenszielen unter besonderer Berücksichtigung } \\
\text { sozial benachteiligter Gruppen } \\
\Rightarrow \text { Stimulation und Förderung von gemeinschaft- } \\
\text { lichen Gesundheitsaktivitäten als Instrument der } \\
\text { Gesundheitsförderung und Prävention }\end{array}$ & $\begin{aligned} \rightarrow & \text { Bonifizierung ergebnisorientierter Kriterien } \\
& \text { (ohne positive Entwicklungshonorierung) } \\
& \text { (Selektionseffekt und Mitnahmeeffekt) } \\
\rightarrow & \text { Auswahl der Kriterien nach marketinstrategischen } \\
\text { Unternehmenszielen mit bewusster Fokussierung } & \text { auf Zielgruppen mit geringen Risiken } \\
\rightarrow & \text { Förderung von Gruppenaktivitäten als Mittel der } \\
& \text { Neukundengewinnung und Kundenbindung }\end{aligned}$ \\
\hline Relation & $\begin{array}{l}\rightarrow \text { Berücksichtigung des Anstrengungswertes bei } \\
\text { der Bewertung der Größendimension } \\
\rightarrow \text { Berücksichtigung von Anreizsteigerungen bei } \\
\text { verhaltensrelevanten Aktivitäten }\end{array}$ & $\begin{array}{l}\rightarrow \text { keine Berücksichtigung des Anstrengungs- } \\
\text { wertes (Verstärkung des Selektionseffekts) } \\
\rightarrow \text { Berücksichtigung von Anreizsteigerungskompo- } \\
\text { nenten mit Bindungscharakter }\end{array}$ \\
\hline Anreize & $\begin{array}{l}\rightarrow \text { Konzentration auf gesundheitsaffine Anreize mit } \\
\text { Elementen zur Steigerung der Chancengleichheit } \\
\rightarrow \text { (freie) Prämien mit gesundheitlichem } \\
\text { Nutzwertcharakter für die Versicherten }\end{array}$ & $\begin{array}{l}\rightarrow \text { Konzentration auf Luxus- und Prestigeanreize mit } \\
\text { Marken- und Eventcharakter } \\
\rightarrow \text { (gebundene) Prämien mit Wettbewerbscharakter } \\
\text { (Kooperationen, Neukundengewinnung/-bindung) }\end{array}$ \\
\hline$=$ Ziel & $\begin{array}{l}\rightarrow \text { Schadensminimierung und -reduzierung guter sowie } \\
\text { schlechter Risikogruppen }\end{array}$ & $\begin{array}{l}\rightarrow \text { Kundenbindung und Neukundengewinnung guter } \\
\text { Risiken, Abwehr von schlechten Risiken }\end{array}$ \\
\hline
\end{tabular}




\section{THEMA}

\section{2.)in welchem Maße sie einen direkten Einfluss auf die} gesundheitliche Zielsetzung ausüben können.

Die Basis bildet eine „gerechte“ Anreizsetzung, eine genaue Zielgruppen- und Bedarfsanalyse sowie eine daraus abgeleitete bedürfnisorientierte Präventionsstrategie. Werden Gerechtigkeits- und Fairnessaspekte nicht adäquat berücksichtigt, so kann dies negative Konsequenzen (z. B. mangelnde Teilnahme, Imageverlust) hervorrufen. Denn bspw. die aktuelle ergebnisabhängige Bonifizierung von Idealzuständen (wie dem BMI, bei ca. 50\% der untersuchten Programme) kann bei den (potentiellen) Teilnehmern nicht nur als unfair und demotivierend empfunden werden, sondern fördert den oft kritisierten Mitnahmeeffekt guter Risiken. Das Gerechtigkeitspostulat ist erfüllt, wenn neben dem Gleichheitsprinzip das „Leistungsprinzip" bei der Anforderungserfüllung (durch Honorierung positiver Entwicklungen im Gesundheitsverhalten etc.) Berücksichtigung findet.[16] Insbesondere bei Risikogruppen besteht die Gefahr, dass Selbstselektionseffekte (auch Kundenselbstselektionswirkungen) zum Tragen kommen, da die Versicherten durch die empfundene Unwahrscheinlichkeit einer Zielerreichung abgeschreckt werden. Bonifizierungskriterien stellen die Teilnehmer - je nach Ausgangslage - vor unterschiedlich hohe Herausforderungen. Besonders Risikogruppen sollten das subjektive Empfinden haben, eine reelle Chance zur aktiven Anforderungsbewältigung leisten zu können. Ist diese Voraussetzung bei verhaltensorientierten (z. B. bei geringer Auswahlmöglichkeit) bzw. ergebnisorientierten Kriterien (z. B. bei einer reinen Orientierung an Idealzuständen) nicht gegeben, so findet eine Selbstselektion bzw. der eigene Ausschluss statt. Angesichts der Komplexität der Thematik besteht im Hinblick auf das Präventionsdilemma erheblicher Forschungsbedarf. Zur Ausschöpfung des Präventionspotenzials gilt es, nicht nur Mitnahmeeffekte guter Risiken zu betrachten, sondern Selbstselektionswirkungen schlechter Risiken verstärkt in das Blickfeld zu nehmen. Als kontraproduktiv für das Gesundheitswesen gelten jene Bonusprogramme, deren Erfolge aufgrund einer marketingstrategischen Ausrichtung lediglich auf Mitnahmeeffekte beruhen, [8] da sie das Solidarprinzip der GKV schwächen. Denn die Reduzierung von Selbstselektionswirkungen schlechter Risiken ist unerlässlich, damit Programme lt. gesetzlicher Vorgabe langfristig überhaupt weitergeführt werden. Im Fall präventiver Bonusprogramme subventionieren die "schlechten Risiken" (bzw. deren positive gesundheitliche Veränderungsprozesse) die "guten Risiken“.

\section{Schlussfolgerung}

Auf Basis der Ergebnisse wird vorgeschlagen, insbesondere das Problem der Selbstselektionswirkungen schlechter Risiken aufgrund unzweckmäßiger Gestaltungen von Bonusprogrammen zu diskutieren. Weiterhin wird zur Diskussion gestellt, ob die Motivation menschlichen Verhaltens basierend auf den Annahmen der Reiz-ReaktionsTheorie tatsächlich in erster Linie extern begründet ist.[17] Auf der Basis des Menschenbildes des Homo oeconomicus wird unterstellt, dass Menschen allein mithilfe monetärer Anreize (extrinsisch) zu steuern und zu beeinflussen sind.[18] Bedürfnisse, Emotionen, Beziehungen und Ängste wie intrinsische Motivation haben hier lediglich eine rudimentäre Bedeutung. Grundsätzlich geht es bei anreizorientierten Präventionsprogrammen darum, in gezielter Weise Wirkungen zu erzeugen, die mit einer hohen Vorhersagewahrscheinlichkeit versehen sind, aber zusätzlichen Schwierigkeiten unterliegen, da die Verhaltensänderung von externen Faktoren (z. B. persönliche Situation) abhängt. Insofern bestimmt nicht nur das „Wollen“, sondern auch das „Können“ die Motivation, respektive dann, wenn es um die Überwindung fest verankerter behavioraler Risikofaktoren mit psychologischen Abhängigkeiten geht. Inwiefern extrinsische Anreize (bzw. Boni) hier als wichtige kurzfristige Treiber fungieren, dass Handlungen überhaupt in Angriff genommen werden, bleibt offen. Je anspruchsvoller die gesundheitliche Verhaltensumstellung (z. B. Nikotinverzicht) ist, desto weniger kann auf intrinsische Motivation langfristig verzichtet werden.[19] Wiederum wird seit einigen Jahren im wissenschaftlichen Diskurs kontrovers diskutiert, inwiefern extrinsische Anreize dazu beitragen, intrinsische Motivation zu schmälern. Da externe Anreize laut Deci mehrere Effekte aufweisen: „Every reward (including feedback) has two aspects, a controlling aspect and an informational aspect which provides the recipient with information about his competence and self-determination. "[20] Auch Bonusprogramme können als kontrollierend oder unterstützend empfunden werden und so intrinsische Motivation zerstören oder fördern. Dieser Effekt wird als Verdrängungseffekt bzw. Verstärkungseffekt bezeichnet. Zusammenfassend kann festgehalten werden, dass unabhängig der Anreizfunktion von Bonusprogrammen wichtige übergreifende Präventionsimpulse ausgehen können - vorausgesetzt bestehenden Informations-, Transformations-, Wissens- und Fähigkeitsbarrieren können zusätzlich überwunden werden. Werden Bonusprogramme allein auf monetäre Größen fixiert, könnte dies langfristig mitunter kontraproduktiv sein, da sie eine Verschwendung knapper Mittel fördern, ohne nachhaltige Effekte zu garantieren (Mitnahmeeffekte). Da fest verankerter behaviorale Risikofaktoren und die Übernahme von Eigenverantwortung nicht alleine durch materielle Anreize erzeugt werden können, steht zur Diskussion in welchem Ausmaß andere Gegebenheiten, wie bspw. die Beziehung zur Krankenkasse, die äußeren Bedingungen, die Attraktivität der Handlung und die Persönlichkeit des Versicherten einen Einfluss auf das Verhalten nimmt. Erste Erkenntnisse auf Basis der gesetzlich geforderten Evaluation stellen Einzelfallstudien dar und können daher lediglich isolierte Lerneffekte bei einzelnen Kassen hervorrufen. Die aktuell differenzierten und isolierten Evaluationen ohne Standardkriterien erschweren eine Vergleichbarkeit und folglich die Generalisierbarkeit möglicher Erfolgsfaktoren. Es wäre wünschenswert, wenn zukünftige Forschungserkenntnisse folglich die Unterschiede und Gemeinsamkeiten einzelner Bonusmodelle berücksichtigen. Gemeinsame Evaluationskriterien und Leitlinien (z. B. auf der Ebene der Spitzenverbände) könnten bspw. helfen, einen Erkenntnisgewinn und eine positive Weiterentwicklung voranzutreiben. Zudem wird 
eine Präzisierung der gesetzlichen Rahmenbedingungen empfohlen.[8]

\section{Literaturverzeichnis}

[1] Lauer T (2004) Bonusprogramm - Rabattsysteme für Kunden erfolgreich gestalten. Springer Verlag, Berlin Heidelberg New York

[2] § 65 a SGB V

[3] §20 SGB V

[4] Lampert T, Saß A Ch, Häfelinger M, Ziese T (2005) Expertise des Robert Koch-Instituts zum 2. Armuts- und Reichtumsbericht der Bundesregierung. Robert-Koch-Institut, Berlin

[5] Sachverständigenrat zur Begutachtung der gesamtwirtschaftlichen Entwicklung: Gesundheitsreform 2006, Gesundheitsreform 2006 - Auszug aus dem Jahresgutachten 2006/07

[6] Ärzte-Zeitung (2007) Nordic Walking auf Mallorca, Wellness an der Ostsee - Marketing-Gag der Kassen oder sinnvolle Prävention? (Meldung vom 22.02.2007)

[7] BKK-BV (2008) Versicherte und Kassen profitieren von Bonusprogrammen (Pressemeldung vom 26.02.2008): http://www.bkk.de/bkk/pressemitteilungen/powerslave,id,421,nodeid,15.html

[8] Knaack N (2007) Chancen und Grenzen der Bonifizierung von Gesundheitsverhalten in der Gesetzlichen Krankenversicherung: eine theoretische und empirische Analyse, Dissertation

[9] Breyer F, Zweifel P, Kifmann M (2005) Gesundheitsökonomie. Springer Verlag, Berlin Heidelberg New York

[10] Höppner K, Buitkamp M, Braun B, Greß St, Rothgang H, Wasem J (2005) Grenzen und Dysfunktionalität des Kassenwettbewerbs in der GKV: Theorie und Empirie der Risikoselektion in Deutschland. Zentrum für Sozialpolitik, Bremen

[11] Die Maxime (soziale Gerechtigkeit und Sicherheit) finden sich im § 1 Abs. 1 SGB V. Da diese schwer konkretisierbar sind, fällt es den Kassen leichter, sich an den Zielen der $\S \S 1$ und 20 SGB V zu orientieren.

[12] Scherenberg V, Greiner W (2008) Präventive Bonusprogramme - Auf dem Weg zur Überwindung des Präventionsdilemma. Hans Huber Verlag, Bern

[13] Spitzenverbände der Krankenkassen (2006) Leitfaden Prävention - Gemeinsame und einheitliche Handlungsfelder und Kriterien der Spitzenverbände der Krankenkassen zur Umsetzung von § 20 Abs. 1 und 2 SGB V

[14] Kossbiel H (1994) Überlegungen zur Effizienz betrieblicher Anreizsysteme. In: Die Betriebswirtschaft, Jg. 94, 1:75-93

[15] Wischer T (2005) Ein Modell zur Beurteilung der Effizienz von Anreizsystemen - Erweiterung des Kossbielschen Effizienzansatzes auf Basis des Rubikon-Modells. Reiner Hampp Verlag, München Mering

[16] Ullrich CG (2004) Sozialpolitische Gerechtigkeitsprinzipien, empirische Gerechtigkeitsüberzeugungen und die Akzeptanz sozialer Sicherungssysteme. In: Liebig St, Lengfeld H, Mau St (Hrsg) Verteilungsprobleme und Gerechtigkeit in modernen Gesellschaften. Campus Verlag GmbH, Frankfurt/Main, S 69-96 [14] Thommen JP, Achleitner AK (2003) Allgemeine Betriebswirtschaft - Umfassende Einführung aus managementorientierter Sicht. Gabler Verlag, Wiesbaden

[17] Frey B (1997) Markt und Motivation. Verlag Franz Vahlen GmbH, München

[18] Falk A (2001) Homo Oeconomicus Versus Homo Reciprocans: Ansätze für ein Neues Wirtschaftspolitisches Leitbild? Working Paper No. 79, Institute for Empirical Research in Economics University of Zurich. Das Modell des Homo oeconomicus lässt wesentliche Determinanten menschlichen Verhaltens unberücksichtigt, da Erwartungen und die Einschätzung des Verhaltens anderer keine Rolle spielen. Der Homo reciprocans belohnt faires Verhalten und bestraft unfaires Verhalten, auch wenn dies für ihn mit materiellen Einbußen verbunden ist. Reziprozität hat nichts mit Altruismus zu tun. Vielmehr entscheidet die empfundene Fairness eines Systems und die Intensität einer Vertrauensbeziehung - neben der eigenen Nutzenabwägung - darüber, wie das eigene Verhalten sein wird.

[19] Frey B, Osterloh M (2002) Managing Motivation. Gabler Verlag, Wiesbaden

[20] Deci E (1980) The Effects of Feedback on Intrinsic Motivation: A Propositional Extension of Cognitive Evaluation Theory from an Organisational Communication Perspective. In: Nimmo D (Hrsg) Communication Yearbook 4. Transaction Publishers, New Brunswick, S 367-387

\section{Europäisches Sozialmodell}

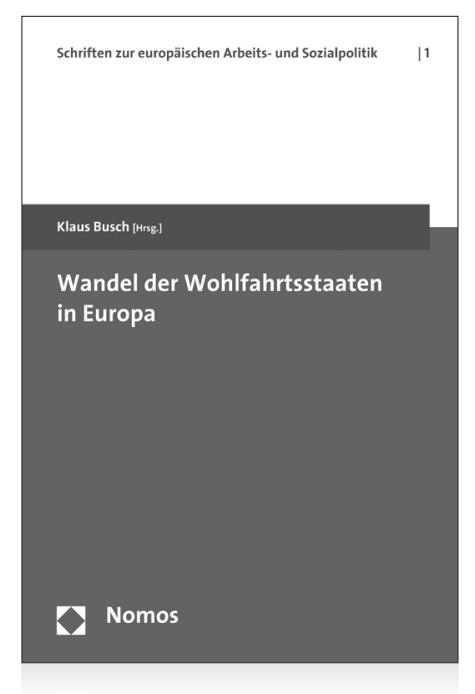

\section{Wandel der Wohlfahrtsstaaten in Europa Herausgegeben von Prof. Dr. Klaus Busch 2008, 298 S., brosch., 54,-€, ISBN 978-3-8329-3123-O (Schriften zur europäischen Arbeits- und Sozial- politik, Bd. 1)}

Die soziale Ungleichheit hat in den letzten 15 Jahren in den Mitgliedstaaten der EU eindeutig zugenommen. In Politik und Wissenschaft ist seither der Ruf nach einem alternativen europäischen Wirtschaftsund Sozialmodell lauter geworden. Der vorliegende Band behandelt den Wandel der betroffenen europäischen Wohlfahrtsstaaten.

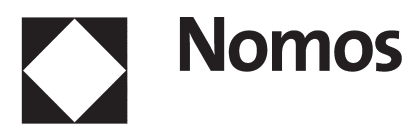

Bitte bestellen Sie bei Ihrer Buchhandlung oder bei Nomos | Telefon 07221/2104-37 | Fax -43 | www.nomos.de| sabine.horn@nomos.de 